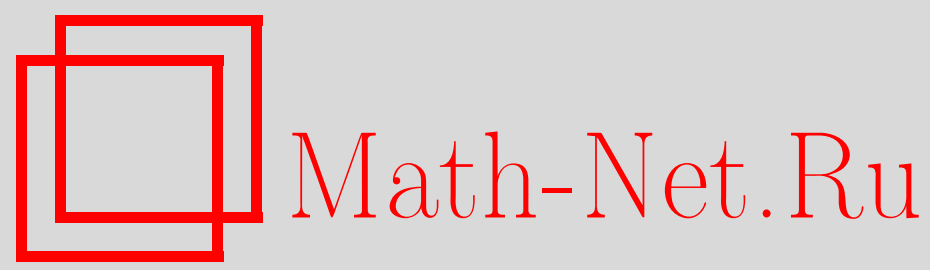

П. А. Крылов, Е. Г. Пахомова, Абелевы группы и регулярные модули, Матем. заметки, 2001, том 69, выпуск 3, 402411

DOI: https://doi.org/10.4213/mzm513

Использование Общероссийского математического портала Math-Net.Ru подразумевает, что вы прочитали и согласны с пользовательским соглашением http://www . mathnet.ru/rus/agreement

Параметры загрузки:

IP : 54.162.27.143

26 апреля 2023 г., 13:25:27

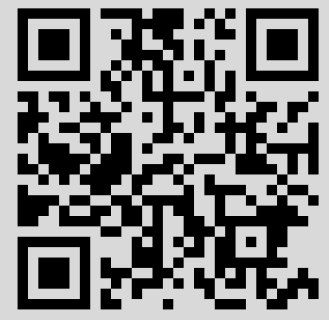




\title{
АБЕЛЕВЫ ГРУППЫ И РЕГУЛЯРНЫЕ МОДУЛИ
}

\author{
П. А. Крылов, Е. Г. Пахомова
}

Рассматривается строение аддитивной группы регулярного модуля. Изучаются абелевы группы, являющиеся регулярными модулями над своими кольцами эндоморфизмов. Описаны нередуцированные эндорегулярные группы и эндорегулярные группы конечного ранга без кручения.

Библиографияя: 18 названий.

В теории абелевых груп активно развивается исследование груп как модулей над их кольцами эндоморфизмов (по поводу этой тематики см. [1], [2]). В данной статье группы рассматриваются как регулярные модули над своими кольцами эндоморфизмов. Такие группы называются эндорегулярными. Регулярный модуль определен в [3, $\S 10.5]$ как модуль, каждый циклический подмодуль которого является прямым слагаемым. Это расширяет понятие регулярного кольца в смысле фон Неймана. В регулярном модуле каждый конечно порожденный подмодуль является прямьп слагаемьм.

Теорема 1 дает определенную информацию об аддитивной группе регулярного модуля. Известной проблемой, примыкающей к теориям абелевых групп и колец, является проблема исследования аддитивных групп колец [4, гл. 17]. Кажется уместным сформулировать более общую задачу, пограничную между теориями абелевых груп и модулей, изучения аддитивных групп модулей (прежде всего из различных специальных классов). Укажем, что в [5] предлагается описать аддитивные группы инъективных модулей. Теорема 1 обобщает теорему 124.1 из книги [4] об аддитивных группах регулярного кольца.

В п. 2 дается описание нередуцированных эндорегулярных групा. Их оказьвается немного. Главньй интерес представляют редуцированные эндорегулярные групшы. Теорема 2 характеризует редуцированные эндорегулярные групшы конечного ранга без кручения.

Модули, встречающиеся в статье, - левые унитарные над ассоциативным кольцом с единицей; групшы - абелевы. Групповые термины, примененные к кольцу или модулю, относятся к их аддитивньп группам; $\oplus, \prod$ - знаки прямой суммы и произведения соответственно; $\mathbb{Q}$ - поле рациональных чисел. Если $A$ - группа, то $E(A)$ - ее кольцо эндоморфизмов, $A_{p}-p$-компонента группы $A$, т.е. наибольшая подгруппа в $A$, являющаяся $p$-группой. При $A_{p} \neq 0$ говорим, что $p$ относится $\kappa A$. Далее, $T(A)-$ периодическая часть группы $A$ - совокупность всех ее элементов конечного порядка; имеем

Работа выполнена при финансовой поддержке Российского фонда фундаментальных исследований, грант № 00-01-00876. 
$T(A)=\bigoplus_{p} A_{p}$. Смешанная групnа - это группа, содержащая как ненулевые элементы конечных порядков, так и элементы бесконечного порядка. Рангом группы без кручения назьвается мощность любой максимальной линейно независимой системы ее элементов. Под рангом без кручения $r_{0}(A)$ смешанной группы $A$ понимается ранг факторгруппы $A / T(A)$. Элементарной группой назьвается прямая сумма циклических групा простых порядков.

1. Аддитивное строение регулярного модуля. Теорема 1 (она анонсирована в [6]) дает довольно ясное представление об аддитивных группах регулярных модулей. Замечание в конце статьи наводит на мысль, что исчерпывающее их описание едва ли достижимо. Приведем ряд простых свойств регулярных модулей.

ЛЕмма 1. Подмодуль регулярного модуля регулярен.

ДоКАЗАТЕЛЬСтво. Пусть $M$ - регулярньй $R$-модуль, $A$ - его подмодуль и $a \in A$. Тогда $M=R a \oplus K$ для некоторого подмодуля $K$. Из $R a \subseteq A$ вьводим $A=R a \oplus(A \cap K)$, что означает регулярность модуля $A$.

Лемма 2. Пусть $h: R \rightarrow S-$ сюрвективный гомоморфизм колеи, $M-S$-модуль. Регулярность $S$-модуля $M$ равносильна его регулярности как притягивающего $R$-модуля.

ДокАЗАТЕЛЬСТво. Достаточно заметить, что $S$-подмодули и $R$-подмодули в $M$ совпадают (см. [7, лемма 11.12]).

Лемма 3. Пусть $M-$ регулярный $R$-модуль, $e^{2}=e \in R$. Тогда еM - регулярный eRe-модуль.

ДокАЗАТЕЛЬСтво. Возьмем произвольный элемент $е m \in e M$. Нужно установить, что $e R e(e m)$ - прямое слагаемое $e R e$-модуля $e M$. Регулярность модуля $M$ влечет $M=$ $R(\mathrm{em}) \oplus K$ для некоторого подмодуля $K$. Откуда $e M=e R e(e m) \oplus e K$.

Теорема 1. Аддитивная группа регулярного $R$-модуля $M$ является прямой суммой некоторой делимой группы без кручения $D$ и редучированной группы $A$ такой, что

$$
\bigoplus_{p} C_{p} \subseteq A \subseteq \prod_{p} C_{p}
$$

где $C_{p}$ - әлементарная $p$-группа, а $A$ сервантна в $\prod_{p} C_{p}$.

ДокАЗАтЕльство. Сначала предположим, что $M$ не имеет кручения как группа. Допустим, напротив, что $p M \neq M$ для некоторого числа $p$. Тогда $p^{2} M \neq p M$. Выберем элемент $x \in p M-p^{2} M$. Ввиду регулярности $M$ имеем $M=R x \oplus L$, где $L$ - какой-то модуль, а $R x \subseteq p M$. Записав $x=p y$ с $y \in M$, найдем $p y \in R x$ и $y \in R x$. Откуда $y \in p M$ и $y=p z$ для некоторого $z \in M$. И далее, $x=p y=p^{2} z \in p^{2} M$, что противоречит выбору элемента $x$.

Пусть теперь $M$ - произвольный регулярньй $R$-модуль. Покажем, что $T(M)$ - элементарная группа. Обозначим через $C_{p} p$-компоненту группы $M$. Допустим напротив, что нашелся элемент $x \in C_{p}$ порядка $p^{k}, k>1$. Имеем $M=R\left(p^{k-1} x\right) \oplus Y$, где $Y-$ некоторый модуль. Запишем $x=z+y$, где $z \in R\left(p^{k-1} x\right), y \in Y$. Откуда находим $0 \neq p^{k-1} x=p^{k-1} z+p^{k-1} y=p^{k-1} y \in Y$, чего не может быть. Таким образом, $T(M)-$ 
элементарная группа. Следовательно, $M=C_{p} \oplus E_{p}$ для каждого $p$, относящегося к $M$, $E_{p}$ - дополнительное слагаемое. Заметим, что $C_{p}$ является подмодулем $R$-модуля $M$. Покажем, что $E_{p}$ - также подмодуль. Поскольку в $E_{p}$ нет элементов порядка $p$, то деление элементов группы $E_{p}$ на $p$ однозначно. Так же, как в первом абзаце доказательства, можно найти, что $p E_{p}=E_{p}$. Отсюда заключаем, что $E_{p}$ - подмодуль. Имеем модульное разложение $M=C_{p} \oplus E_{p}$ для каждого $p \in P$, где $P$ обозначает множество всех простых чисел, относящихся к $M$.

Для каждого $p \in P$ пусть $e_{p}$ - проекция модулей $M \rightarrow C_{p}$ с ядром $E_{p}$. С помощью этих проекций строим модульный гомоморфизм

$$
f: M \rightarrow \prod_{p \in P} C_{p}
$$

полагая $f(m)=\left\langle e_{p}(m)\right\rangle$ для каждого $m \in M$. Ясно, что

$$
\operatorname{ker} f=\bigcap_{p \in P} E_{p}
$$

Обозначим подмодуль $\operatorname{ker} f$ через $D$. Тогда $D$ - регулярньй модуль без кручения (лемма 1). По уже доказанному $D$ - делимая группа. Значит, можно написать прямую сумму $M=D \oplus A$ для какой-то группы $A$.

Возьмем фактормодуль $M / D$, изоморфньй $\operatorname{im} f$. Удобно отождествить $M / D \mathrm{c} \operatorname{im} f$. Так как $f$ действует на сумме $\bigoplus_{p} C_{p}$ тождественно, то можем считать, что

$$
\bigoplus_{p} C_{p} \subseteq M / D \subseteq \prod_{p} C_{p}
$$

где вложения являются модульными. Убедимся в сервантности подгруппы $M / D$ в $\prod_{p} C_{p}$. Это равносильно делимости факторгруппы $(M / D) / T(M / D)$, которую обозначим буквой $G$. Заметим, что $T(M / D)=\bigoplus_{p} C_{p}$. Если $p \in P$, то $G$ делится на $p$ как гомоморфный образ $p$-делимой группы $M / C_{p}$ (она изоморфна $E_{p}$ ). Если же $p \notin P$, т.е. $M$ не имеет $p$-компоненты, то $p M=M$ (см. замечание о $p$-делимости групшы $E_{p}$ выше). Тем более делится на такое $p$ группа $G$. Итак, $G$ - делимая группа, а $M / D$ - сервантная подгруппа в $\prod_{p} C_{p}$. Так как $A \cong M / D$, то для группы $A$ имеем сервантные вложения

$$
\bigoplus_{p} C_{p} \subseteq A \subseteq \prod_{p} C_{p}
$$

Таким образом, $M=D \oplus A$, где $D$ и $A$ такие, как утверждается в теореме. Теорема доказана.

Неясно, можно ли подгрупш $A$ выбрать так, чтобы она была подмодулем. Тем не менее, в ситуации теоремы 1 имеет место такой результат.

СлЕДСТВИЕ 1. 1) $M / D$ - регулярный $R$-модуль.

2) Если модуль $D$ конечно порожсден, то $A$ является подмодулем. 
ДокаЗАтЕльство. 1) Пусть элемент $\bar{a}=a+D \in M / D, a \in A$. Тогда $M=R a \oplus X$ для некоторого подмодуля $X$. Проверим, что $M / D=R \bar{a} \oplus \bar{X}$, где $R \bar{a}=(R a+D) / D$, $\bar{X}=(X+D) / D$. Нужно лишь убедиться, что $R \bar{a} \cap \bar{X}=0$ или $(R a+D) \cap(X+D) \subseteq D$. Если $b \in(R a+D) \cap(X+D)$, то $b=r a+c=x+d$, где $r \in R, c, d \in D, x \in X$. Поскольку $D$ - вполне инвариантньй подмодуль как максимальная делимая подгруппа, то $D=(D \cap R a) \oplus(D \cap X)$. Запишем $c=c_{1}+c_{2}, d=d_{1}+d_{2}$, где $c_{1}, d_{1} \in D \cap R a$, $c_{2}, d_{2} \in X$. Теперь получаем $r a+c_{1}-d_{1}=x+d_{2}-c_{2} \in R a \cap X=0$ и $r a=d_{1}-c_{1} \in D$. Отсюда $b=r a+c \in D$. Итак, $M / D=R \bar{a} \oplus \bar{X}$ и $M / D$ - регулярный модуль.

2) Конечно порожденньй подмодуль $D$ выделяется в $M$ прямым слагаемым в модульном смысле. Следствие доказано.

Пусть, как в теореме, $M=D \oplus A$. Групшы $A$ и $M / D$ канонически изоморфны, поэтому $A$ можно превратить в $R$-модуль, полагая для $r \in R$ и $a \in A, r$ о $a=a_{1}$, где $r a=d+a_{1}, d \in D, a_{1} \in A$. При этом $A \cong M / D$ как $R$-модули. По следствию $1 A-$ регулярньй $R$-модуль при указанном действии $R$ на $A$.

Вопрос Ричмена и Уокера [5] об описании аддитивных групп инъективных модулей остается открытым. Напомним об одном замечательном факте: инъективньй модуль обязательно алгебраически компактен как абелева группа [8, с. 209].

СлЕДСТВИЕ 2. На әруппе $M$ можно задать регулярный инбективный модуль тогда и только тогда, когда $M=\prod_{p} C_{p} \oplus D$, где $C_{p}$ - әлементарная $p$-группа, $D$ - делимая группа без кручения.

ДокАЗАТЕЛьСтво. Пусть $M$ - регулярньй инъективный модуль. Тогда $M=A \oplus D$, где $A$ и $D$ - такие группы, как в теореме 1 . Группа $A$ алгебраически компактна как прямое слагаемое алгебраически компактной группы. Из $\bigoplus_{p} C_{p} \subseteq A \subseteq \prod_{p} C_{p}$, сервантности $A$ в $\prod_{p} C_{p}$ и определения алгебраически компактной групшы $[8, \S 38]$ выводится, что в действительности $A=\prod_{p} C_{p}$. Обратно, пусть $M=\prod_{p} C_{p} \oplus D$. Тогда $C_{p}-$ регулярный инъективный $F_{p}$-модуль $\left(F_{p}\right.$ - поле из $p$ элементов), $D$ - регулярный инъективный $\mathbb{Q}$-модуль, а $M$ будет регулярным инъективньгм модулем над кольцом $\prod_{p} F_{p} \times \mathbb{Q}$.

2. Эндорегулярные нередуцированные группы. Применим теорему $1 \mathrm{k}$ описанию групा, указанных в заглавии.

ЛЕмма 4. Прямое слагаемое әндорегулярной группы есть әндорегулярная групna.

ДокАЗАТЕЛЬСтво. Пусть $G$ - эндорегулярная групша, $A$ - ее прямое слагаемое, $e: G \rightarrow A-$ проекция. Тогда $A=e G, E(A)=e E(G) e$ и осталось сослаться на леммy 3.

ПРЕДЛОЖЕНИЕ 1. Нередуцированная группа $G$ является әндорегулярной тогда и только тогда, когда $G=D \oplus A$, где $D$ - делимая группа без кручения, $A$ әлементарная группа (мохет быть, $A=0$ ).

ДокАЗАтЕЛЬСтво. Пусть $G$ - эндорегулярная групша. Тогда $G=D \oplus A$, где $D$ и $A-$ групшы из теоремы 1 . Согласно условию $D \neq 0$. Известное строение делимых групп позволяет заключить, что $D$ - циклический $E(G)$-модуль. В таком случае $A-$ $E(G)$-подмодуль, т.е. вполне характеристическая подгруппа в $G$ (следствие 1$)$. Допустим, что $A \neq 0$ и элемент $a \in A$ имеет бесконечньй порядок. Существует мономорфизм 
$\langle a\rangle \rightarrow D$, которьй продолжается в силу инъективности групшы $D$ до гомоморфизма $A \rightarrow D$. Но это противоречит вполне характеристичности $A$ в $G$. Значит, $A-$ периодическая группа, $A=\bigoplus_{p} C_{p}$, где $C_{p}$ - элементарная $p$-групша. Таким образом, $A-$ элементарная группа.

Допустим, что $G=\bigoplus_{p} C_{p} \oplus D$, где $C_{p}$ - элементарная $p$-группа, $D$ - делимая группа без кручения. Все групшы $C_{p}$ и $D$ являются неприводимьми $E(G)$-модулями, а $G-$ вполне приводимый $E(G)$-модуль. Тем более, $G$ - регулярный $E(G)$-модуль.

ЗАмЕЧАниЕ 1. Эндорегулярность нередуцированной групшы $G$ равносильна также тому, что $E(G)$ - регулярное кольцо [4, предложение 112.7].

ЗАмЕчАниЕ 2. Некоторая группа $G$ является вполне приводимым $E(G)$-модулем тогда и только тогда, когда $G=D \oplus A$, где $D$ - делимая без кручения, $A$ - элементарная групшы.

Работа [5] содержит описание групп, инъективных над своими кольцами эндоморфизмов, т.е. эндоинъективных групш. Из [5], следствия 2 и предложения 1 получается такой результат.

СлЕДСТВИЕ 3. Группа $G$ является әндорегулярной әндоинвективной тогда и только тогда, когда она имеет один из следующих видов:

1) $G=D \oplus A$, әде $D$ - делимая без кручения, $A$ - әлементарная группь конечных рангов;

2) $G=\prod_{p} C_{p}$, где $C_{p}-$ конечная әлементарная $p$-группа для каждого $p$.

3. Эндорегулярные $s p$-группы. Предложение 1 сводит проблему описания эндорегулярньх групп к случаю редуцированных групп. Согласно теореме 1 для редуцированной эндорегулярной групшы $A$ имеют место сервантные вложения

$$
\bigoplus_{p} A_{p} \subseteq A \subseteq \prod_{p} A_{p}
$$

где $A_{p}$ обозначает $p$-компоненту группы $A$. Группа $\bigoplus_{p} A_{p}$ эндорегулярна (предложение 1). Поэтому считаем, что $A \neq \bigoplus_{p} A_{p}$, т.е. $A$ - смешанная групша. Такие смешанные группы, лежащие между суммой и произведением своих $p$-компонент, привлекли в последнее время внимание многих специалистов [9]-[14]. Здесь мы назьваем их $s p$-группами. Дадим точное определение: $s p$-группой называется редуцированная смешанная группа $A$ с бесконечным числом ненулевых $p$-компонент такая, что вложение $\bigoplus_{p} A_{p} \rightarrow A$ продолжается до сервантного вложения $A \rightarrow \prod_{p} A_{p}$. Таким образом, для $s p$-групшы $A$ можно считать, что $\bigoplus_{p} A_{p} \subset A \subseteq \prod_{p} A_{p}$, причем $A$ сервантна в $\prod_{p} A_{p}$. Здесь и далее подразумевается, что $p$ пробегает множество всех простых чисел, относящихся к $A$. Элементарная $s p$-груnпа-это такая $s p$-группа, все $p$-компоненты которой - элементарные групшы. В свете сказанного вьше получается, что при изучении эндорегулярньх групп можно ограничиться элементарньпи $s p$-группами.

Приведем несколько фактов о $s p$-группах. Они будут нужны для доказательства основной теоремы 2. Для групшы $A$ положим $E_{t}(A)=\operatorname{Hom}(A, T(A))$ и $E_{f}(A)=\{\alpha \in E(A) \mid$ $\alpha A$ содержится в сумме конечного числа компонент $\left.A_{p}\right\}$. Здесь $E_{t}(A)$ и $E_{f}(A)$ - идеалы кольца $E(A)$, причем $E_{f}(A) \subseteq E_{t}(A)$. 
Напомним определение категории Уокера Walk [15]. Объектами категории Walk служат группы, а множество морфизмов $\operatorname{Hom}_{W}(A, B)$ есть $\operatorname{Hom}(A, B) / \operatorname{Hom}(A, T(B))$. Walk является аддитивной категорией с бесконечными суммами и ядрами. Заметим, что $E(A) / E_{t}(A)$ - это кольцо эндоморфизмов группы $A$ в Walk. Обозначим его $E_{W}(A)$.

Пусть $W$ - полная подкатегория категории Walk, состоящая из $s p$-групп $A$ таких, что $E_{t}(A)=E_{f}(A)$. Из $[15]$ выводится эквивалентность следуюших утверждений:

1) $A \cong B$ в $W$;

2) $A \oplus T \cong B \oplus S$, где $T$ и $S$-периодические грушш с конечным числом $p$-компонент;

3) $A \cong C \oplus S, B \cong C \oplus T$, где $C \in W, S$ и $T$ такие, как в 2 ).

Говорят, что идемпотенты расщепляются в аддитивной категории $\mathscr{E}$, если всякий раз когда $e^{2}=e \in E_{\mathscr{E}}(A)$, то сушествуют объект $B \in \mathscr{E}$ и морфизмы $g \in \operatorname{Hom}_{\mathscr{E}}(B, A)$, $h \in \operatorname{Hom}_{\mathscr{E}}(A, B)$ с $g h=e$ и $h g=1_{B}$ [16, определение 18.15].

Для групшы $A$ фиксируем обозначения: $R=E(A), R_{t}=E_{t}(A)$ и $S=R / R_{t}$.

\section{Лемма 5. В категории $W$ расщепляются идемпотенты.}

ДоказАтельство. Пусть даны группа $A \in W$ и идемпотент $\bar{e}=e+R_{t}$ кольца $S$, где $e \in R$. Имеем $e^{2}=e+r$ для некоторого $r \in R_{t}$. Можно записать $A=C \oplus V$, где $r C=0, V$-прямая сумма конечного числа $p$-групा, причем $C$ и $V$ не имеют $p$-компонент для одинаковых $p$. Определим $f \in R$, полагая $f(x)=e(x)$ для $x \in C$ и $f V=0$. Тогда $f^{2}=f$ и $A=B \oplus \operatorname{ker} f$, где $B=f A$. Если $g: B \rightarrow A$ - вложение, $h: A \rightarrow B-$ проекция, то $h g=1_{B}, g h=f$. Откуда $\bar{h} \bar{g}=1, \bar{g} \bar{h}=\bar{f}=\bar{e}$ (черта обозначает смежный класс относительно $R_{t}$ ). Итак, нашлись группа $B \in W$ и морфизмы $\bar{g}, \bar{h}$ с указанньми свойствами. Значит, $\bar{e}$ расщепляется.

Лемма 6. Если $A \in W$, то всякое конечное полное ортогональное множество идемпотентов кольца $S$ можно поднять до полного ортогонального множества идемпотентов кольиа $R$.

ДокАЗАТЕЛЬСТво. Как видно из доказательства леммы 5 , множество идемпотентов кольца $S$ сразу можно взять в виде $e_{1}+R_{t}, \ldots, e_{n}+R_{t}$, где $e_{1}, \ldots, e_{n}$ - идемпотенты кольца $R$. Для любых различных индексов $i, j$ имеем $e_{i} e_{j} \in R_{t}$. Поэтому существует разложение $A=C_{i j} \oplus V_{i j}$ такое, что $e_{i} e_{j} C_{i j}=0, V_{i j}$ - прямая сумма конечного числа $p$-групп. При этом $C_{i j}$ и $V_{i j}$ не имеют $p$-компонент для одинаковых $p$. Пусть $V$-сумма всех групा $V_{i j}, C$ - дополнительное к $V$ слагаемое, $A=C \oplus V$. Считаем, что все $e_{i}$ аннулируют $V$. Тогда $e_{1}, \ldots, e_{n}$ - система ортогональных идемпотентов кольца $R$. Полнота в $S$ означает, что $\left(e_{1}+R_{t}\right)+\cdots+\left(e_{n}+R_{t}\right)=1$. Добавляя к $V$ некоторые $p$-компоненты группы $C$, можно добиться того, что $e_{1}+\cdots+e_{n}=1$ в $R$. Лемма доказана.

Понятие групповой суммы в $W$ имеет теоретико-групповю интерпретацию. Из предыдущего (см. также $[17$, лемма 7.1$])$ можно вывести следуюший результат.

СлеДСтвие 4. Группа $A$ является прямой суммой групп $A_{1}, \ldots, A_{n}$ в $W$ тогда и только тогда, когда $A=B_{1} \oplus \cdots \oplus B_{n}$, где $B_{i} \in W$ u $A_{i} \cong B_{i}$ в $W, i=1, \ldots, n$.

Поскольку $W$ - аддитивная категория с расщепляюшимися идемпотентами, в ней выполняется теорема Крулля-Шмидта [16, теорема 18.18]. Справедливы также стандартные соотношения между группой $A$ из $W$ и ее кольцом эндоморфизмов $E_{W}(A)$. Так, следующее предложение можно доказать непосредственно с помощью леммы 6 и аналогичного факта для модулей (см. [18, § 3.4, лемма 1$])$. 


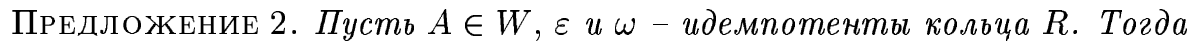

1) справедлив изоморфизм групп $\operatorname{Hom}_{W}(\omega A, \varepsilon A) \cong \bar{\varepsilon} \bar{\omega}$ и кольчевой изоморфизм $E_{W}(\varepsilon A) \cong \bar{\varepsilon} S \bar{\varepsilon}$ (черта обозначает смежсный класс относительно $\left.R_{t}\right)$;

2) әквивалентны следующие утверждения:

а) существуют $r, s \in S$ такие, ито $r s=\bar{\varepsilon} u s r=\bar{\omega}$;

б) $\varepsilon A \cong \omega A$ в $W$;

в) $S \bar{\varepsilon} \cong S \bar{\omega}$ как левье $S-м о д у л и$.

Пусть $A \in W$. В дополнение к введенньп уже обозначениям положим $T=T(A)$, $V=A / T$. Тогда $V$ - $\mathbb{Q}$-пространство, $S$ - $\mathbb{Q}$-алгебра. Кроме того, $V$ - левьй $R$-модуль, аннулятор которого равен $R_{t}$. Поэтому $V$ является левым $S$-модулем: $s v=r v$, где $v \in V, r \in R, s=r+R_{t}$.

Обозначим через $W_{f}$ полную подкатегорию категории $W$, состоящую из групा с конечньм рангом без кручения. Пусть $A \in W_{f} . Q$-алгебра $S$ вкладывается в $\operatorname{End}_{Q} V$, и поэтому $S$ - конечномерная алгебра. Используя лемму 6, получаем, что неразложимость группы $A$ в $W_{f}$ равносильна локальности кольца $S$. Следовательно, $A$ обладает свойством единственности разложения в категории $W_{f}$ в прямую сумму неразложимых в $W_{f}$ групш [10] (см. замечание вьше о теореме Крулля-Шмидта в $W$ ). Из предложения 2 , опираясь на известное строение артинова полупростого кольца, выводится такой результат (он получается также из [12, теорема 2.4]).

СлЕдСтвИЕ 5. Записанные ниже утверждения о группе $A \in W_{f}$ әквивалент$H b l$ :

1) $V$ - вполне приводимый $S$-модуль;

2) $S$ - полупростая алгебра;

3) $A=\bigoplus_{i=1}^{n} A_{i}$, где $\operatorname{Hom}\left(A_{i}, A_{j}\right)=\operatorname{Hom}\left(A_{i}, T\left(A_{j}\right)\right)$ для всех различных $i, j=$ $1, \ldots, n ; A_{i}=\bigoplus_{k=1}^{n_{i}} A_{i k}, A_{i k} \cong A_{i l}$ в Wu $E_{W}\left(A_{i k}\right)-\operatorname{mело~}(i=1, \ldots, n$, $\left.k, l=1, \ldots, n_{i}\right)$.

ПРЕДЛОЖЕНИЕ 3. Пусть $A$ - әлементарная sp-группа конечного ранга без кручения. Тогда $A=C \oplus E$, где $C \in W_{f}, E$ - әлементарная группа.

ДокАЗАтЕльство. Для каждого $p$, относящегося к $A$, запишем $A=A_{p} \oplus B_{p}$, где $B_{p}$ - дополнительное слагаемое. Пусть $e_{p}$ обозначает проекцию $A \rightarrow A_{p}$ с ядром $B_{p}$. Выберем свободную подгруппу $F$ группы $A$ с образующими $c_{1}, \ldots, c_{n}$, где $n$ равно рангу без кручения групшы $A$. Любая подгруппа элементарной группы является прямьп слагаемым. Следовательно, для каждого $p$ имеем $A_{p}=e_{p} F \oplus E_{p}$, где $E_{p}$ - некоторая подгруппа. Положим $\bar{A}=\prod_{p} A_{p}$. Группу $A$ можно считать сервантной подгруппй в $\bar{A}$. Тогда $\bar{A}=X \oplus Y$, где $X=\prod_{p} e_{p} F, Y=\prod_{p} E_{p}$. Обозначив $C=A \cap X$ и $E=\bigoplus_{p} E_{p}$, проверим, что $A=C \oplus E$. По построению $C \cap X=0$ и $T=T(A) \subset C+E$. Из $(C+E) / T=((X+T) / T) \cap(A / T)$ заключаем, что подгруппа $(C+E) / T$ сервантна в $A / T$. Кроме того, она содержит базис $c_{1}+T, \ldots, c_{n}+T \mathbb{Q}$-пространства $A / T$. Следовательно, $A=C \oplus E$. Имеем $A_{p}=C_{p} \oplus E_{p}=e_{p} F \oplus E_{p}$. Поэтому ограничениепроекции $e_{p}$ на $C$ будет проекцией $C \rightarrow C_{p}$. Так как $C_{p}=e_{p} F$, то группа $C$ удовлетворяет так назьваемому условию на проекции и по [11, следствие 2.4] $C \in W_{f}$. 
Лемма 7. Пусть дана группа $A=C \oplus E$, где $E$ - әлементарная группа. Тогда әндорегулярность группы $A$ әквивалентна әндорегулярности группы $C$.

ДокАЗАТЕЛьСтво. Допустим, что группа $C$ эндорегулярна. Возьмем элемент $a \in$ $A, a=c+e$, где $c \in C, e \in E$. Имеем $R a=(R a \cap C) \oplus(R a \cap E)$, где $R a \cap C(R a \cap E)-$ вполне характеристическая подгруппа в $C$ (в $E)$. Причем, $R a \cap E$ совпадает с суммой некоторых $p$-компонент $E_{p}$. Справедливо также равенство $R a \cap C=E(C) c+\operatorname{Hom}(E, C) e$. Здесь $E(C) c$ - прямое слагаемое $E(C)$-модуля $C$ на основании эндорегулярности группы $C$. Группа $\operatorname{Hom}(E, C) e$ является суммой конечного числа некоторых $p$-компонент $C_{p}$ группы $C$. Отсюда $R a \cap C$-прямое слагаемое групшы $C$, a $R a \cap E$-прямое слагаемое группы $E$. Поэтому $R a$ - прямое слагаемое групшы $A$. Дополнительное к $R a$ слагаемое будет модульньм, т.е. оно является вполне характеристической подгрупой. Это вытекает из таких соображений. Согласно теореме 1 все $A_{p}$ - элементарные групш и, значит, они будут модульными слагаемыми в $A$. Затем $R a$ содержит все те компоненты $A_{p}$, относительно которых элемент $a$ имеет ненулевые координаты. Итак, $A$ - эндорегулярная группа. Обратное содержится в лемме 4. Лемма доказана.

Используем введенные ранее обозначения: $T=T(A), V=A / T, R=E(A), R_{t}=$ $\operatorname{Hom}(A, T)$ и $S=R / R_{t}$. По аналогии с группами без кручения назовем $s p$-группу $A$ неприводимой, если $V$ - неприводимьй $S$-модуль.

Пусть $A-s p$-группа, $a \in A$. Для каждого $p$ имеем разложение $A=A_{p} \oplus B_{p}$. Запишем $a=a_{p}+b_{p}$, где $a_{p} \in A_{p}, b_{p} \in B_{p}$. Элемент $a_{p}$ назьваем $p$-координатой әлемента $а$. Введем также следующие множества простых чисел: $\pi(A)=\left\{p \mid A_{p} \neq 0\right\}$ и $\pi(a)=\{p \mid a$ имеет ненулевую $p$-координату $\}$.

Теорема 2. Элементарная sp-группа $A$ конечного ранга без кручения әндорегулярна тогда и только тогда, когда $A=C_{1} \oplus \cdots \oplus C_{n} \oplus E$, где $C_{1}, \ldots, C_{n}$ - неприводимые sp-группь, причем $\operatorname{Hom}\left(C_{i}, C_{j}\right)=0$ для любых различных $i, j=1, \ldots, n$, E- әлементарная группа.

ЗАмечание 3 . Условие $\operatorname{Hom}\left(C_{i}, C_{j}\right)=0$ эквивалентно тому, что $C_{i}$ и $C_{j}$ имеют ненулевые $p$-компоненты для различньх $p$.

ДокАЗАТЕЛЬСтво. Предположим, что группа $A$ эндорегулярна. Убедимся, что $V-$ регулярньй $S$-модуль. Возьмем некоторьй элемент $\bar{a}=a+T \in V$, отличньй от нуля. Ввиду эндорегулярности групшы $A$ имеем $A=R a \oplus B$ для какого-то $R$-модуля $B$. Покажем, что $V=S \bar{a} \oplus \bar{B}$, где $\bar{B}=(B+T) / T$. Ясно, что $S \bar{a}$ и $\bar{B}$ порождают $V$. Допустим, что $\overline{r a}=\bar{b} \in S \bar{a} \cap \bar{B}$. В групше $A$ получаем $b=r a+c$ для некоторого $c \in T$. Можно записать $c=s a+d$, где $s \in R, d \in T(B)$. Теперь находим $b-d=r a+s a \in R a \cap B=0$, и далее, $b=d \in T, \bar{b}=0$. Значит, $V=S \bar{a} \oplus \bar{B}$ и $V$ - регулярньй $S$-модуль. Артиновость кольца $S$ (абзац перед следствием 5 ) влечет вполне приводимость $S$-модуля $V[3$, с. 266 , упр. 16]. Точность этого модуля в свою очередь дает полупростоту кольца $S$.

По предложению $3 A=C \oplus E$, где $C \in W_{f}, E$ - элементарная группа. Имеем $S=E(C) / E_{t}(C)=E_{W}(C)$ и, значит, $E_{W}(C)$ - полупростое артиново кольцо. По следствию 5 можно написать $A=C_{1} \oplus \cdots \oplus C_{n} \oplus E$, где $\operatorname{Hom}\left(C_{i}, C_{j}\right)=\operatorname{Hom}\left(C_{i}, T\left(C_{j}\right)\right)$ для всех $i, j$ с $i \neq j$. Кроме того, если обозначить $R_{i}=E\left(C_{i}\right), S_{i}=R_{i} / E_{t}\left(C_{i}\right)$, то $S_{i}$-простое артиново кольцо. Установим, что каждая $C_{i}$ - неприводимая $s p$-группа. Обозначим $V_{i}=C_{i} / T\left(C_{i}\right)$. Заметим, что $S=S_{1} \times \cdots \times S_{n}, V=V_{1} \oplus \cdots \oplus V_{n}$ (при естественном отождествлении). Нужно доказать неприводимость $S_{i}$-модуля $V_{i}$. Допустим напротив, 
что этот модуль не является неприводимьп. Найдется элемент $\bar{a}=a+T \in V_{i}$, для которого $S_{i} \bar{a} \neq V_{i}$. По лемме 4 группа $C_{i}$ эндорегулярна, поэтому $C_{i}=R_{i} a \oplus M$, где

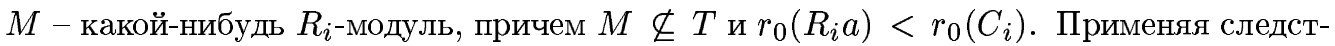
вие 5 к групше $C_{i}$, получаем $C_{i}=B_{1} \oplus \cdots \oplus B_{m}$, где $B_{1} \cong B_{2} \cong \cdots \cong B_{m}$ в категории $W$ и все $B_{i}$ неразложимы в $W$. Далее, $R_{i} a=\left(R_{i} a \cap B_{1}\right) \oplus \cdots \oplus\left(R_{i} a \cap B_{m}\right)$, где $R_{i} a \cap B_{1} \cong \cdots \cong R_{i} a \cap B_{m}$ в $W$. Так как $r_{0}\left(R_{i} a\right)<r_{0}\left(C_{i}\right)$, то $r_{0}\left(R_{i} a \cap B_{1}\right)<r_{0}\left(B_{1}\right)$. Но это невозможно по свойству изоморфизма прямых разложений в $W_{f}$ (замечание перед следствием 5$)$. Таким образом, $V_{i}$ - неприводимый $S_{i}$-модуль, а $C_{i}$ - неприводимая группа.

Возьмем некоторые слагаемые $C_{i}$ и $C_{j}(i \neq j)$ и допустим, что $\operatorname{Hom}\left(C_{i}, C_{j}\right) \neq 0$. Если $C_{i}$ и $C_{j}$ имеют лишь конечное число $p$-компонент для одинаковых $p$, то все эти компоненты можно присоединить к $E$. После чего будет $\operatorname{Hom}\left(C_{i}, C_{j}\right)=0$. Поэтому пусть $C_{i}$ и $C_{j}$ имеют ненулевые $p$-компоненты для бесконечного числа одних и тех же $p$. Выберем

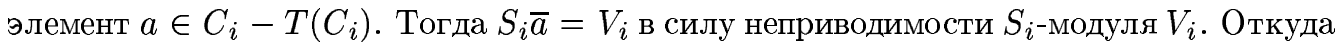
$C_{i} / R_{i} a-$ периодическая групша. Поскольку $C_{i}$ - эндорегулярная группа (лемма 4 ), то $C_{i}=R_{i} a \oplus G$, где $G$ - элементарная группа с конечным числом $p$-компонент (нужно учесть, что $\left.C_{i} \in W\right)$. Эндорегулярность же группы $A$ влечет $A=R a \oplus B$ для некоторой группы $B$. Подгруппа $R a$ вполне характеристична в $A$, поэтому из разложения $A=C_{1} \oplus \cdots \oplus C_{n} \oplus E$ вьводим $R a=\left(R a \cap C_{j}\right) \oplus M$, где $M$ - дополнительное слагаемое. Так как $\operatorname{Hom}\left(C_{i}, C_{j}\right)=\operatorname{Hom}\left(C_{i}, T\left(C_{j}\right)\right)$, то понятно, что $R a \cap C_{j} \subseteq T\left(C_{j}\right)$ и $R a \cap C_{j}$ - элементарная группа. Кроме того, $R a \cap C_{j}$ - прямое слагаемое групшы $C_{j}$; $p$-координаты элемента $a$ отличны от нуля для почти всех $p$, относяшихся к $C_{i}$. Ввиду нашего соглашения о $p$-компонентах групп $C_{i}$ и $C_{j}$ получаем, что $a$ имеет ненулевые $p$-координаты для бесконечного множества чисел $p$, относящихся к $C_{j}$. Можно сделать вывод, что элементарная группа $R a \cap C_{j}$ имеет бесконечно много $p$-компонент. Однако это противоречит тому обстоятельству, что $A \in W$. Таким образом, $\operatorname{Hom}\left(C_{i}, C_{j}\right)=0$.

Предположим теперь, что вьполнены условия теоремы. В силу леммы 7 достаточно установить эндорегулярность суммы $C_{1} \oplus \cdots \oplus C_{n}$. Из $\operatorname{Hom}\left(C_{i}, C_{j}\right)=0$ при $i \neq j$ в свою очередь вьводим, что нужно убедиться в эндорегулярности каждой из групп $C_{1}, \ldots, C_{n}$. Возьмем, например, $C_{1}$. Неприводимость $S_{1}$-модуля $V_{1}$ влечет, что для любых элементов $b, c \in C_{1}-T\left(C_{1}\right)$ существуют $\varphi, \psi \in R_{1}$ и $g, h \in T\left(C_{1}\right)$ такие, что $c=\varphi b+g$ и $b=\psi c+h$ (здесь, конечно, $R_{1}=E\left(C_{1}\right), S_{1}=R_{1} / E_{t}\left(C_{1}\right)$ и $\left.V_{1}=C_{1} / T\left(C_{1}\right)\right)$. Заключаем, что множества $\pi(b)$ и $\pi(c)$ "почти" равны в том смысле, что оба множества $\pi(b)-\pi(c)$ и $\pi(c)-\pi(b)$ конечны. Пусть элементы $v_{1}+T\left(C_{1}\right), \ldots, v_{k}+T\left(C_{1}\right)$ составляют базис $\mathbb{Q}$-пространства $C_{1} / T\left(C_{1}\right)$. Как только что замечено, $\pi\left(v_{i}\right)-\pi\left(v_{j}\right)$ - конечное множество для любых $i$ и $j$. Теперь пусть элемент $a \in C_{1}$. Если $a \in T\left(C_{1}\right)$, то понятно, что $R_{1} a$ есть сумма $p$-компонент групшы $C_{1}$ для всех $p \in \pi(a)$. Значит, $R_{1} a$ - прямое слагамое $R_{1}$-модуля $C_{1}$. Если $a \in C_{1}-T\left(C_{1}\right)$, то элемент $a$ равен линейной комбинации элементов $v_{1}, \ldots, v_{k}$ с рациональными коэффициентами плюс некоторьй элемент из $T\left(C_{1}\right)$. Из всего сказанного можно сделать вьвод, что $\pi(a)$ состоит из почти всех чисел множества $\pi\left(C_{1}\right)$, т.е. $\pi\left(C_{1}\right)-\pi(a)$ - конечное множество. Запишем $C_{1}=B \oplus G$, где $G$ - сумма $p$-компонент групшы $C_{1}$ для всех $p \in \pi\left(C_{1}\right)-\pi(a)$. Очевидно, что $a \in B$,

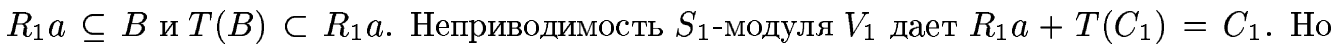
$T\left(C_{1}\right)=T(B) \oplus G$ и $C_{1}=R_{1} a+T\left(C_{1}\right)=R_{1} a \oplus G$. Итак, $R_{1} a$ является $R_{1}$-модульным прямым слагаемым в $C_{1}$, что доказывает эндорегулярность группы $C_{1}$. Теорема доказана. 
Класс эндорегулярных $s p$-групп бесконечного ранга без кручения, по-видимому, мало обозрим. Авторы располагают примером эндорегулярной $s p$-групшы $A$ бесконечного ранга без кручения, для которой $A / T(A)$ - суперразложимьй регулярньй $R / R_{t}$-модуль.

\section{СПИСОК ЦИТИРОВАННОЙ ЛИТЕРАТУРЫ}

[1] Марков В.Т., Михалев А. В., Скорняков Л. А., Туганбаев А. А. Колца эндоморфизмов модулей и структуры подмодулей // Итоги науки и техники. Алгебра. Топология. Геометрия. Т. 21. М.: ВИНИТИ, 1983. С. 183-254.

[2] Крылов П.А. Абелевы группы без кручения как модули над своими кольцами эндоморфизмов // Абелевы группы и модули. Вып. 13, 14. Томск: Изд-во ТГУ, 1996. С. 77-104.

[3] Каш Ф. Модули и кольца. М.: Мир, 1981.

[4] Фукс Л. Бесконечные абелевы группы. Т. 2. М.: Мир, 1977.

[5] Richman F., Walker E. A. Modules over PIDs that are injective over their endomorphism rings // Ring Theory. New York: Acad. Press, 1972. P. 363-372.

[6] Пахомова Е. Г. Абелевы группы как регулярные модули над кольцами эндоморфизмов // Межд. конф. "Всесибирские чтения по математике и механике". Т. 1. Томск, 1997. С. 32-33.

[7] Фейс К. Алгебра: кольца, модули и категории. Т. 1. М.: Мир, 1977.

[8] Фукс Л. Бесконечные абелевы группы. Т. 1. М.: Мир, 1974.

[9] Glaz S., Wickless W. Regular and principal projective endomorphism rings of mixed abelian groups // Comm. Algebra. 1994. V. 22. № 4. P. 1161-1176.

[10] Wickless W. A functor from mixed groups to torsion-free groups // Contem. Math. 1995. V. 171. P. 407-419.

[11] Albrecht U. F., Goeters H. P., Wickless W. The flat dimension of mixed abelian groups as E-modules // Rocky Mount. J. Math. 1995. V. 25. № 2. P. 569-590.

[12] Albrecht U. Mixed abelian groups with artinian quasi-endomorphism ring // Comm. Algebra. 1997. V. 25. № 11. P. 3497-3511.

[13] Fomin A., Wickless W. Self-small mixed abelian groups $G$ with $G / T(G)$ finite rank divisible // Comm. Algebra. 1998. V. 26. №11. P. 3563-3580.

[14] Крылов П. А. Кольца эндоморфизмов смешанных абелевых групп // Межд. конф. "Всесибирские чтения по математике и механике". Т. 1. Томск, 1997. С. 20-21.

[15] Warfield R.B.(Jr.) The structure of mixed abelian groups // Lect. Notes Math. 1997. V. 616. P. 1-38.

[16] Фейс К. Алгебра: кольца, модули и категории. Т. 2. М.: Мир, 1979.

[17] Arnold D. M. Finite rank torsion-free abelian groups and rings // Lect. Notes Math. 1982. V. 931.

[18] Ламбек И. Кольца и модули. М.: Мир, 1971.

Томский государственный университет

Поступило

E-mail: krylov@math.tsu.ru

12.07.1999 